\title{
A Decade of Active Surveillance in the PRIAS Study : An Update and Evaluation of the Criteria Used to Recommend a Switch to Active Treatment
}

\section{The PRIAS study group}

2016

The PRIAS study group 2016 , ' A Decade of Active Surveillance in the PRIAS Study : An Update and Evaluation of the Criteria Used to Recommend a Switch to Active Treatment ' , European Urology , vol. 70 , no. 6 , pp. 954-960 . https://doi.org/10.1016/j.eururo.2016.06.007

http://hdl.handle.net/10138/231341

https://doi.org/10.1016/j.eururo.2016.06.007

publishedVersion

Downloaded from Helda, University of Helsinki institutional repository.

This is an electronic reprint of the original article.

This reprint may differ from the original in pagination and typographic detail.

Please cite the original version. 


\title{
A Decade of Active Surveillance in the PRIAS Study: An Update and Evaluation of the Criteria Used to Recommend a Switch to Active Treatment
}

\author{
Leonard P. Bokhorst ${ }^{a, *}$, Riccardo Valdagni $^{b}$, Antti Rannikko $^{c}$, Yoshiyuki Kakehi $^{d}$, \\ Tom Pickles ${ }^{e}$, Chris H. Bangma ${ }^{a}$, Monique J. Roobol ${ }^{a}$, \\ for the PRIAS study group ${ }^{1}$

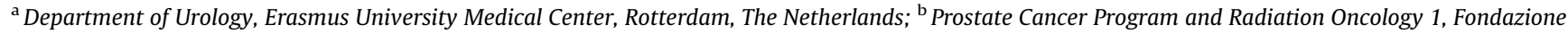 \\ IRCSS Istituto Nazionale dei Tumori, Milan, Italy; ' Department of Urology, Helsinki University Central Hospital, Helsinki, Finland; ${ }^{\mathrm{d}}$ Department of Urology, \\ Kagawa University Faculty of Medicine, Kagawa, Japan; ${ }^{\mathrm{e}}$ Department of Radiation Oncology, British Columbia Cancer Agency, Vancouver, Canada
}

\section{Article info}

Article history:

Accepted June 7, 2016

Associate Editor:

James Catto

\section{Keywords:}

Prostatic neoplasms

Active surveillance

Prostate-specific antigen

Prostate biopsy

Disease progression

MRI

\begin{abstract}
Background: The Prostate Cancer Research International Active Surveillance (PRIAS) study was initiated a decade ago to study the most optimal selection and follow-up of men on active surveillance (AS).

Objective: We report on $10 \mathrm{yr}$ of follow-up of men on AS in the PRIAS study and evaluate if criteria used to recommend a switch to active treatment truly predict unfavorable outcome on subsequent radical prostatectomy (RP).

Design, setting, and participants: Men with low-risk prostate cancer were included and followed prospectively on AS. Follow-up consisted of regular prostate-specific antigen (PSA) tests, digital rectal examinations, and biopsies. Men with Gleason $>3+3$, more than two positive biopsy cores, or stage higher than CT2 were advised to switch to active treatment (until 2014, a PSA doubling time [PSA DT] of 0-3 yr was also used).

Outcome measurements and statistical analysis: Reclassification rates, treatment after discontinuation, and outcome on RP after discontinuing AS were reported. Regression analysis on the outcome of RP was used to evaluate the predictive value of criteria currently used to recommend a switch to active treatment. Kaplan-Meier and competing risk analysis were used to report discontinuation rates over time and long-term oncologic end points.

Results and limitations: A total of 5302 men were included in PRIAS across 18 countries. Reclassification rates remained stable on all subsequent biopsies, with 22-33\% of men having either Gleason $>3+3$ or more than two positive cores on any repeat biopsy. At 5 and $10 \mathrm{yr}$ of follow-up, $52 \%$ and $73 \%$ of men, respectively, had discontinued AS, most of them because of protocol-based reclassification. A third of men undergoing subsequent RP had favorable pathologic tumor features (Gleason $3+3$ and pT2). Of the criteria used to recommend a switch to active treatment, more than two positive cores and a PSA DT of $0-3 \mathrm{yr}$ were not predictive of unfavorable pathologic outcome on RP.

Conclusions: A substantial group of men discontinued AS without subsequent unfavorable tumor features on RP; therefore, we propose Gleason upgrading and CT3 as the only indicators for an immediate switch to active treatment. Surrogate indicators (eg, more than two positive cores and a fast-rising PSA) should not trigger immediate active treatment but rather further investigation to confirm the suspicion of higher risk disease.

Patient summary: We confirmed the safety of active surveillance as a treatment option for men with low-risk prostate cancer; however, some changes could be made to the follow-up protocol to safely increase the number of men who remain on active surveillance.
\end{abstract}

(C) 2016 European Association of Urology. Published by Elsevier B.V. All rights reserved.

1 See Supplement 1 for a list of study group members.

* Corresponding author. Department of Urology, Erasmus University Medical Center, Room NA-1710, PO Box 2040, 3000 CA Rotterdam, The Netherlands Tel. +31 10703 2243; fax: +31 107035315. E-mail address: l.bokhorst@erasmusmc.nl (L.P. Bokhorst). 


\section{Introduction}

The Prostate Cancer Research International Active Surveillance (PRIAS) study was initiated a decade ago (2006) with the aim of providing evidence-based recommendations on how to select and follow men with low-risk prostate cancer (PCa) on active surveillance (AS) [1]. Other than most single (academic) center AS studies, the PRIAS study aims to represent a more real-world situation with inclusions from academic, nonacademic, and private practices across the world, greatly increasing the generalizability of the results. Since its introduction, the PRIAS study has developed into the largest prospective AS study worldwide, with at present $>150$ participating centers in 18 countries. Data on the first 500 study participants were reported in 2010 [2], with an update on 2500 men in 2012 [3].

We report on $>5000$ men followed on AS in the PRIAS study to date, and we specifically evaluate the criteria used to recommend a switch to active treatment by assessing their ability to predict outcome on radical prostatectomy $(\mathrm{RP})$ in men discontinuing AS.

\section{Methods}

All centers prospectively enter data on inclusion, and they follow up through the PRIAS Web site (www.prias-project. org) that automatically provides recommendations on follow-up [1]. The original criteria for inclusion are Gleason score $\leq 3+3$, stage not higher than cT2c, PSA $\leq 10 \mathrm{ng} / \mathrm{ml}$, two or fewer cores positive for PCa, prostate-specific antigen (PSA) density $\leq 0.2 \mathrm{ng} / \mathrm{ml}$ per cubic centimeter, and fitness for curative treatment. In 2012 and 2015, inclusion criteria were adapted to include minimal Gleason $3+4$ and accommodate changes in the number of positive cores caused by biopsies targeted by magnetic resonance imaging (MRI) or saturation biopsies (all changes made to the study protocol are summarized in Table 1). No minimum number of biopsy cores is required, but based on prostate volume the following is advised: $<40 \mathrm{~cm}^{3}: 8$ cores; $40-60 \mathrm{~cm}^{3}: 10$ cores; and $>60 \mathrm{~cm}^{3}: 12$ cores ( $>80 \%$ complied).

In the first $2 \mathrm{yr}$ of follow-up, a PSA test is scheduled every $3 \mathrm{mo}$, and a digital rectal examination (DRE) is scheduled every 6 mo. Thereafter, PSA is measured every 6 mo, and DRE is performed once yearly. Standard repeat biopsies are scheduled $1,4,7$, and $10 \mathrm{yr}$ after diagnosis and subsequently every $5 \mathrm{yr}$. Yearly biopsies are only recommended if PSA doubling time (PSA DT) is between 0 and $10 \mathrm{yr}$. A bone scan is recommended if the PSA level is $\geq 20 \mathrm{ng} / \mathrm{ml}$.

Criteria used to recommend a switch to active treatment are Gleason $>3+3$, more than two positive cores, and stage higher than CT2. A PSA DT between 0 and $3 \mathrm{yr}$ (if at least four PSA values are available) was used to recommend immediate active treatment until the end of 2014 but was dropped afterward due to the low number of men complying with this recommendation and the high percentage of men receiving unnecessary treatment, as described in a recent publication [4]. Criteria used to recommend a switch to active treatment were adapted for those included with Gleason $3+4$ and more than two cores based on MRI or saturation biopsies (Table 1 ).

More information on the follow-up schedule and a flowchart of the current follow-up protocol can be found online (www.prias-project.org). Follow-up for the current analysis ended in November 2015.

\subsection{Statistical analysis}

Descriptive statistics were used to report baseline characteristics, reclassification rates on subsequent biopsies, treatment after discontinuation, and outcome on RP after discontinuing AS. Pathologic outcome on RP was divided in three categories: favorable pathology (Gleason $3+3$ and pT2), intermediate pathology (Gleason $3+4$ and pT2), and unfavorable pathology (Gleason $\geq 4+3$ or pT3 or higher),

Table 1 - Changes made and proposed to the PRIAS study protocol

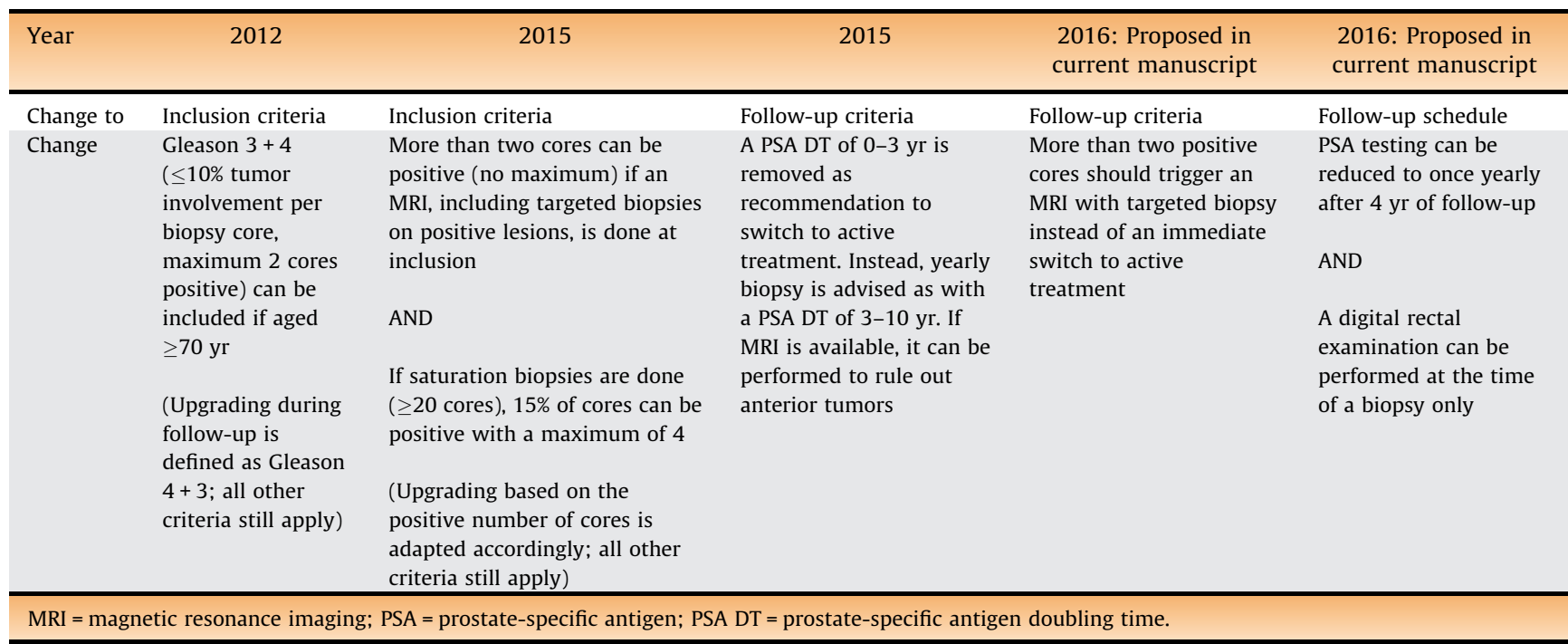




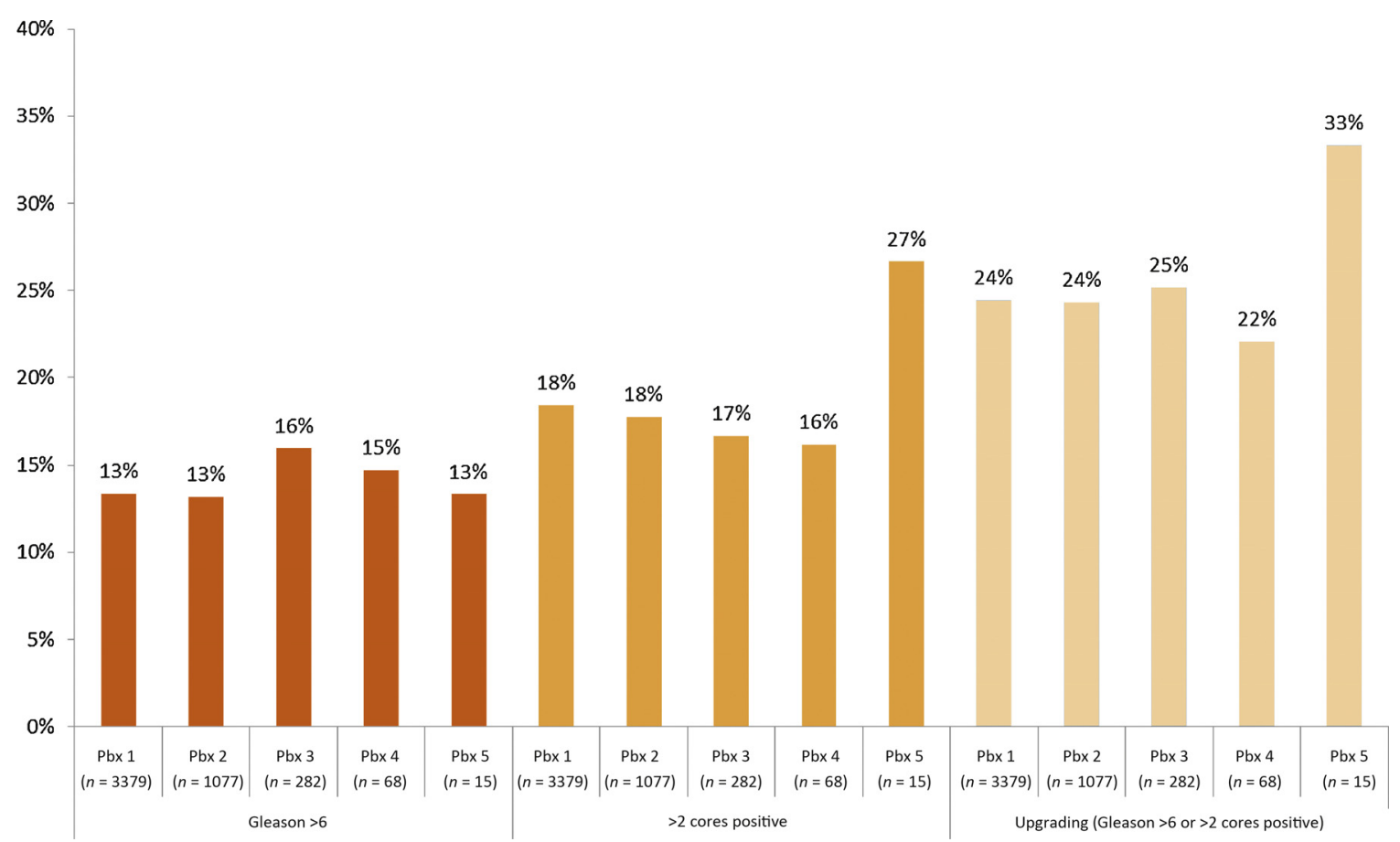

Fig. 1 - Reclassification rates on subsequent repeat biopsies during follow-up. $\mathrm{Pbx}=$ repeat biopsy.

based on a previous analysis and recent reports on low metastatic potential of men with Gleason $3+3$ organconfined disease on RP [5-7].

Multinomial logistic regression analysis was used to evaluate the predictive value of criteria used to recommend a switch to active treatment (Gleason $>3+3$, more than two positive cores, PSA DT between 0 and $3 \mathrm{yr}$ ) on pathologic RP outcome. The predictive value of cT3 could not be assessed due to the low number of men with this characteristic. Corrections were made for clinical characteristics at the time of switching to active treatment (age and PSA).

Kaplan-Meier and competing risk analysis were used to report discontinuation rates over time, (PCa) mortality, and a combined end point of biochemical recurrence (BCR; defined as a PSA level $\geq 0.2 \mathrm{ng} / \mathrm{ml}$ after RP or a PSA level $2.0 \mathrm{ng} / \mathrm{ml}$ above the nadir after radiation therapy [RT]) or local recurrence after active treatment (either RP or RT), metastasis, and PCa death, whichever occurred first for all men included.

Separate analyses were done for all men included and for men fulfilling the original inclusion criteria, except for the multinomial analysis that was only done in men fulfilling the original inclusion criteria. For analysis, SPSS for Windows v.21.0 (IBM Corp., Armonk, NY, USA) and the survival package of R (R Foundation for Statistical Computing, Vienna, Austria) were used.

\section{Results}

A total of 5302 men were included and followed prospectively on AS in the PRIAS study across 18 countries (Supplementary Fig. 1). Of these men, 622 were followed on AS $>5 \mathrm{yr}$ and 107 were followed for $>7.5 \mathrm{yr}$. At diagnosis, the median age was $65.9 \mathrm{yr}$, the median PSA was $5.7 \mathrm{ng} / \mathrm{ml}$, and most men had one positive biopsy core (69\%) with Gleason $3+3$ (99\%) and a clinical stage T1c (88\%) (Table 2). A total of 216 men (4\%) did not fully comply with the

Table 2 - Characteristics at diagnosis of all men included in the PRIAS study

\begin{tabular}{|c|c|}
\hline Characteristic & Result \\
\hline Age, yr & $65.9(61.0-70.4)$ \\
\hline PSA, ng/ml & $5.7(4.5-7.1)$ \\
\hline Prostate volume, $\mathrm{cm}^{3}$ & $45(35-59)$ \\
\hline PSA density, $\mathrm{ng} / \mathrm{ml}$ per $\mathrm{cm}^{3}$ & $0.13(0.09-0.16)$ \\
\hline No. of biopsy cores & $12(10-12)$ \\
\hline \multicolumn{2}{|l|}{ No. of cores positive } \\
\hline 1 & $3643(69)$ \\
\hline 2 & $1615(30)$ \\
\hline$\geq 3$ & $44(1)$ \\
\hline \multicolumn{2}{|l|}{ Gleason } \\
\hline $3+3$ & $5271(99)$ \\
\hline $3+4$ & $31(1)$ \\
\hline \multicolumn{2}{|l|}{ T stage } \\
\hline cT1c & $4649(88)$ \\
\hline cT2a & $579(11)$ \\
\hline cT2b & $54(1)$ \\
\hline cT2c & $20(<1)$ \\
\hline \multicolumn{2}{|l|}{ Charlson score } \\
\hline 0 & $3745(71)$ \\
\hline 1 & $264(5)$ \\
\hline 2 & $734(14)$ \\
\hline$\geq 3$ & $559(11)$ \\
\hline Total & $5302(100)$ \\
\hline
\end{tabular}


Table 3 - Discontinuation and treatment after discontinuation

\begin{tabular}{|c|c|c|c|c|c|c|c|c|}
\hline & $\mathrm{RP}$ & RT & HT & WW & Other & Unknown & Died/lost to FU & Total \\
\hline \multicolumn{9}{|l|}{ Non-protocol based } \\
\hline Anxiety/Patient request (\%) & $52(29)$ & $32(18)$ & $2(1)$ & - & $2(1)$ & $89(50)$ & - & $177(100)$ \\
\hline Other/Unknown (\%) & $108(45)$ & $78(32)$ & $2(1)$ & - & $27(11)$ & $27(11)$ & - & $242(100)$ \\
\hline Switch to WW & - & - & - & $134(100)$ & - & - & - & $134(100)$ \\
\hline Died & - & - & - & - & - & - & $57(100)$ & $57(100)$ \\
\hline Lost to FU & - & - & - & - & - & - & $56(100)$ & $56(100)$ \\
\hline Protocol based (\%) & $496(45)$ & $419(38)$ & $29(3)$ & $30(3)$ & $30(3)$ & $98(9)$ & - & $1102(100)$ \\
\hline Total (\%) & $656(37)$ & $529(30)$ & $33(2)$ & $164(9)$ & $59(3)$ & $214(12)$ & $113(6)$ & $1768(100)$ \\
\hline
\end{tabular}

original inclusion criteria (due to the changes in protocol or because the PRIAS Web site currently allows off-protocol inclusions). Subanalyses in men fulfilling the original inclusion criteria yielded identical results and thus are not presented.

During follow-up, 3379 men received at least one repeat biopsy; 1077 men received two biopsies, 282 men had three biopsies, 68 men had four biopsies, and 15 men had five biopsies. Reclassification rates remained stable on all subsequent biopsies, with 13-16\% of men having Gleason $>3+3,16-27 \%$ of men having more than two cores positive for PCa, and $22-33 \%$ of men having either Gleason $>3+3$ or more than two positive cores (Fig. 1).

A total of 1768 of the 5302 men discontinued AS until the end of follow-up, most of them $(n=1102)$ because of protocol-based reclassification. Treatment after discontinuation was RP or RT in $67 \%$ of men, and only $3 \%$ received hormonal therapy as primary treatment (Table 3 ). There were no differences in tumor characteristics (PSA, PSA DT, Gleason, and number of positive cores on last biopsy) between men switching to RP or RT, but the latter had a 2-yr higher median age at the time of discontinuation (67 vs $69 \mathrm{yr}$, respectively; $p<0.001$ ).
After 5 and $10 \mathrm{yr}$ of follow-up, respectively, $48 \%$ and $27 \%$ of men were still on AS, $34 \%$ and $41 \%$ discontinued because of protocol-based reclassification, $5 \%$ and $5 \%$ discontinued due to anxiety/patient request (without having reclassification, anxiety and patient request were equally distributed), $5 \%$ and $15 \%$ switched to watchful waiting (WW) or died of another cause (without having reclassification), and 8\% and $12 \%$ discontinued for other reasons (without having reclassification) (Fig. 2).

Pathology data for 360 men receiving RP after discontinuing AS were available for analysis. For men who switched to RP due to anxiety, 13 (57\%) had a favorable pathologic outcome, 6 (26\%) had an intermediate pathologic outcome, and $4(17 \%)$ had an unfavorable pathologic outcome. For men who switched to RP because of protocol-based reclassification, 82 (30\%) had a favorable pathologic outcome, 85 (34\%) had an intermediate pathologic outcome, and 100 (36\%) had an unfavorable pathologic outcome. Overall, pT3a, pT3b, pT4, Gleason $\geq 8$, and N1 were found in $61,13,2,14$, and 1 (of 119 men receiving a lymph node dissection), respectively. Large differences in the distribution of outcomes were observed between different protocol-based reasons to discontinue AS (Table 4). On regression

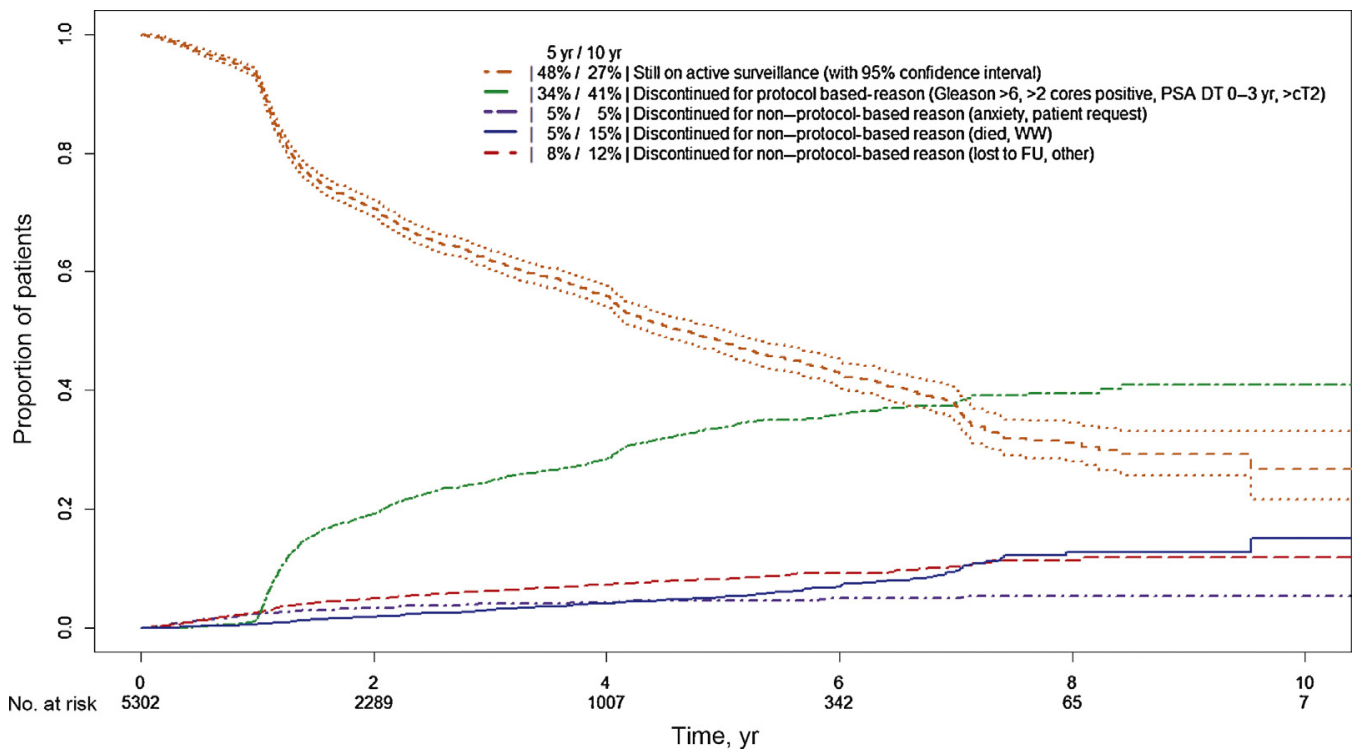

Fig. 2 - Discontinuation of active surveillance over time, stratified by reason for discontinuation.

FU = follow-up; PSA DT = prostate-specific antigen doubling time; $W W=$ watchful waiting. 
Table 4 - Outcome on radical prostatectomy after discontinuing active surveillance

\begin{tabular}{|c|c|c|c|c|}
\hline & $\begin{array}{c}\text { Favorable } \\
\text { (Gleason } 3+3 \text { and pT2) }\end{array}$ & $\begin{array}{c}\text { Intermediate } \\
\text { (Gleason } 3+4 \text { and pT2) }\end{array}$ & $\begin{array}{c}\text { Unfavorable } \\
\text { (Gleason } \geq 4+3 \text { or } \geq \mathrm{pT} 3 \text { ) }\end{array}$ & Total \\
\hline \multicolumn{5}{|l|}{ Non-protocol based, $n$ (\%) } \\
\hline Anxiety/Patient request & $13(57)$ & $6(26)$ & $4(17)$ & $23(100)$ \\
\hline Other/Unknown & $28(47)$ & $12(20)$ & $20(33)$ & $60(100)$ \\
\hline \multicolumn{5}{|l|}{ Protocol based, $n$ (\%) } \\
\hline \multicolumn{5}{|l|}{ 1. Only Gleason $>3+3$} \\
\hline Gleason $3+4$ & $7(27)$ & $15(58)$ & $4(15)$ & $26(100)$ \\
\hline Gleason $\geq 4+3$ & $1(7)$ & $3(21)$ & $10(71)$ & $14(100)$ \\
\hline 2. Only $>2$ cores positive & $28(41)$ & $22(32)$ & $18(26)$ & $68(100)$ \\
\hline 3. Only PSA DT $0-3$ yr & $24(46)$ & $9(17)$ & $19(37)$ & $52(100)$ \\
\hline 4. Only cT3 & $1(50)$ & - & $1(50)$ & $2(100)$ \\
\hline Combination $1+2$ & $1(2)$ & $28(55)$ & $22(43)$ & $51(100)$ \\
\hline Combination $1+3$ & $2(18)$ & $3(27)$ & $6(55)$ & $11(100)$ \\
\hline Combination $2+3$ & $16(50)$ & $6(19)$ & $10(31)$ & $32(100)$ \\
\hline Combination $1+2+3$ & $2(10)$ & $9(43)$ & $10(48)$ & $21(100)$ \\
\hline Total, $n(\%)$ & $123(34)$ & $113(31)$ & $124(34)$ & $360(100)$ \\
\hline
\end{tabular}

Table 5 - Predictors of intermediate (Gleason $3+4$ and pT2) and unfavorable outcome (Gleason $\geq 4+3$ or $\geq$ pT3) on radical prostatectomy"

\begin{tabular}{|c|c|c|c|c|}
\hline & \multicolumn{2}{|c|}{ Intermediate } & \multicolumn{2}{|c|}{ Unfavorable } \\
\hline & OR $(95 \% \mathrm{CI})$ & $p$ value & OR $(95 \% \mathrm{CI})$ & $p$ value \\
\hline Age at discontinuation, $\mathrm{yr}$ & $1.04(0.99-1.09)$ & 0.136 & $1.04(0.99-1.09)$ & 0.101 \\
\hline Last PSA, ng/ml & $0.93(0.85-1.03)$ & 0.146 & $1.00(0.91-1.09)$ & 0.925 \\
\hline PSA DT $0-3$ yr & $0.71(0.37-1.38)$ & 0.312 & $1.44(0.79-2.63)$ & 0.230 \\
\hline No. of positive cores $>2$ on last biopsy & $1.50(0.83-2.70)$ & 0.183 & $1.37(0.78-2.43)$ & 0.274 \\
\hline Gleason $>6$ on last biopsy & $7.44(3.68-15.06)$ & $<0.001$ & $6.12(3.04-12.32)$ & $<0.001$ \\
\hline
\end{tabular}

analysis, only Gleason score $>6$ on the last biopsy was a statistically significant predictor of intermediate or unfavorable pathologic outcome on RP (Table 5).

Until the end of follow-up of all men included, 30 men had biochemical recurrence (BCR) after active treatment (either RP or RT), 10 men had local recurrence, 8 developed metastasis, and 1 died of PCa, resulting in $98 \%$ and $94 \%$ of all men included to be free of BCR, local recurrence, metastasis, and PCa death at 5 and $10 \mathrm{yr}$ after diagnosis, respectively (Supplementary Fig. 2). Other-cause mortality and diseasespecific mortality for all men included were $3 \%$ and $<1 \%$, respectively, at $5 \mathrm{yr}$ after diagnosis and $11 \%$ and $<1 \%$, respectively, at $10 \mathrm{yr}$ after diagnosis (Supplementary Fig. 3).

\section{Discussion}

AS aims to reduce the overtreatment of tumors that are very unlikely to cause symptoms if left untreated. Nevertheless, the possibility remains that tumors with initial low-risk features will turn out not to be indolent and develop into lethal disease. AS is supposed to filter out these men selectively as soon as possible while avoiding invasive treatment in most cases that prove to be truly indolent. The first part of the aim of AS seems partially fulfilled. In the Toronto and Johns Hopkins cohorts of men on AS, very low PCa mortality and metastasis rates were observed, comparable with those after more invasive treatment (RP and RT)
$[8,9]$. Although only a few men were followed $>10 \mathrm{yr}$ in the current analysis, results support the safety of AS. PCa mortality was $<1 \%$, and a combined end point of adverse outcome (BCR, local recurrence, metastasis, or PCa death) was observed in only $6 \%$ of men $10 \mathrm{yr}$ from diagnosis.

In the current analysis, we found only $50 \%$ of men to still be on AS after 5 yr of follow-up and approximately only $25 \%$ after $10 \mathrm{yr}$ of follow-up, lower than reported by other AS studies [8-10]. Some of these differences could be explained by the setup of the PRIAS study, which is not a strictly controlled single academic center study but instead tries to represent a real-world situation (eg, resulting in more men switching to active treatment without a clear protocolbased reason or more men switching to WW). Nevertheless, even if accounting for the $15 \%$ of men stopping AS because of a switch to WW or other cause mortality, a substantial number of men (60\% of the initial cohort) received a form of active treatment after $10 \mathrm{yr}$ of follow-up.

This finding, together with the observation that a third of men still had a favorable pathologic outcome on RP, indicates that the criteria used to recommend a switch to active treatment are far from optimal. On regression analysis, the only statistically significant predictor of intermediate or unfavorable pathologic outcome on RP was a Gleason score $>6$ on last biopsy. In men who switched to RP due to more than two cores positive on last biopsy and in men with a PSA DT of 0-3 yr, the rates having favorable 
pathologic outcome were close to $50 \%$, although the rates of unfavorable pathologic outcome were higher than in men who switched to active treatment without a protocol-based recommendation. Both of these protocol-based indications seem to increase the risk of unfavorable pathologic outcome but are not specific enough to trigger an immediate switch to active treatment. Importantly, PSA DT 0-3 and more than two cores positive with PCa together are responsible for $>50 \%$ of all recommendations to switch to active treatment.

To achieve a higher rate of men who continue AS while selectively identifying those with unfavorable disease, we propose a change of protocol for the PRIAS study. Instead of an immediate switch to active treatment if more than two cores are positive, men should receive further investigation to confirm higher risk disease. Because MRI is shown to have a negative predictive value for Gleason upgrading very close to $100 \%$ in men on AS [11-13], it currently seems the best method to exclude the presence of a higher Gleason score. If the MRI is negative, AS can be continued; if a lesion is present, targeted biopsies should confirm Gleason upgrading before a switch to active treatment is advised. MRI with targeted biopsies in men with increased risk is expected to detect most of the men with truly unfavorable tumor characteristics $[11,14]$. This modification is in line with the recently changed recommendation in men with a PSA DT of 0-3 yr and with the changed criteria that allow inclusion of any number of positive cores if an MRI with targeted biopsies is done [4]. Gleason $>6$ and cT3 will thus remain the only indicators for an immediate switch to active treatment. It is estimated, based on the data in this paper, that because of the suggested protocol change instead of 43 of 100 men, 64 of 100 men could have stayed on AS after three repeat biopsies.

In the (near) future, MRI might even be able to replace systematic repeat biopsies altogether. Systematic biopsies currently appear one of the largest burdens for men on AS $[4,15,16]$, and in fact they are redundant in three-quarters of men because they do not show reclassification (Fig. 1). But before we can definitively adopt such a change, we must collect enough data on men with a negative MRI who simultaneously received systematic biopsies. We issue a plea for increased inclusion of men in the PRIAS MRI side study (www.prias-project.org) to further establish the negative predictive value of MRI in men on AS. If confirmed, many systematic biopsies can be replaced by less invasive imaging.

Some changes to the number and frequency of follow-up visits remain to be discussed. PSA testing is done regularly and is used to calculate PSA DT (used to recommend more frequent biopsies) and a bone scan (if PSA $>20 \mathrm{ng} / \mathrm{ml}$ ). We previously showed data from the PRIAS study indicating that after 4 yr of follow-up, both a change in PSA DT triggering a biopsy and an absolute PSA $>20$ occurred very infrequently [17]. Clinical utility was low (all bone scans were negative and biopsies were only advanced by $6 \mathrm{mo}$ ). It was concluded that PSA testing can be reduced to once yearly after $4 \mathrm{yr}$ of follow-up.

It is sometimes suggested to use an absolute PSA value (eg, $>20 \mathrm{ng} / \mathrm{ml}$ ) to recommend a switch to active treatment.
The PRIAS study did not include such a recommendation because it was felt that once included with a PSA $\leq 10 \mathrm{ng} / \mathrm{ml}$, the PSA value could only rise slowly (in which case benign prostatic hyperplasia might be a more likely cause) or fast, but then PSA DT would trigger further investigation with biopsies to exclude rapid tumor development as its cause. The analysis presented in Table 5 confirms that this initial assumption is now justified because the absolute PSA value does not show a positive correlation with RP outcome within the current follow-up protocol that includes regular repeat biopsies and additional biopsies in the case of a fast rising PSA. We therefore do not recommend an absolute PSA cut-off to discontinue AS.

Because reclassification on DRE only occurred in 10 men ( $<1 \%$ of all reclassifications), one could also argue for reducing the number of DREs, for example, performing a DRE only at the time of a biopsy, although the potential benefit of reducing this relative inexpensive and easy-toperform test could be questioned. The largest benefit should come from individualizing the frequency of repeat biopsies (or in the future possibly MRIs). Currently we individualize the biopsy frequency only based on the PSA DT; however, several other predictors of reclassification were specified (eg, PSA density, the number of positive biopsy cores, and time since last biopsy) $[3,4,18,19]$. Risk prediction models were already developed and should be validated and updated in several cohorts to prolong the time to next biopsy in men with a low risk of reclassification and increase the frequency in men with high risk [19]. In the future, these models can be supplemented by newly validated markers predictive of outcome [20].

Such models can also be used to assist in the timing of when to stop AS and switch to WW. Simultaneous predictions of life expectancy and time until symptoms from a low-risk tumor left untreated are needed. This is one of the topics of the Movember-GAP3 project [21], which combines most of the worldwide available AS cohorts including the PRIAS study.

Criticisms of the current analysis are that follow-up data of men who discontinued AS, including outcome of RP, were missing in several cases. Although limiting the power of some of the analyses, there was no indication of selective reporting that could have affected the results (men with and without RP data available did not differ in terms of age, PSA, PSA DT, Gleason, and number of positive cores on last biopsy). Longer follow-up would more firmly establish the low rates of metastasis and PCa deaths.

\section{Conclusions}

After a decade of AS in the PRIAS study, criteria used to recommend a switch to active treatment do not seem selective enough to avoid unnecessary switches to active treatment. A substantial proportion of men abandoning AS based on the advice of a protocol do not have unfavorable features after RP. We propose Gleason score upgrading or cT3 on DRE as the only reasons for a direct switch to active treatment. Other factors, such as more than two positive biopsy cores and fast-rising PSA, should first trigger 
further investigation to confirm the suspicion of higher risk disease.

Author contributions: Leonard P. Bokhorst had full access to all the data in the study and takes responsibility for the integrity of the data and the accuracy of the data analysis.

Study concept and design: Bokhorst, Roobol, Rannikko, Valdagni, Pickles, Kakehi, Bangma.

Acquisition of data: Bokhorst, Roobol, Rannikko, Valdagni, Pickles, Kakehi, Bangma.

Analysis and interpretation of data: Bokhorst, Roobol.

Drafting of the manuscript: Bokhorst.

Critical revision of the manuscript for important intellectual content: Bokhorst, Roobol, Rannikko, Valdagni, Pickles, Kakehi, Bangma.

Statistical analysis: Bokhorst, Roobol.

Obtaining funding: None.

Administrative, technical, or material support: None.

Supervision: Bokhorst, Roobol, Rannikko, Valdagni, Pickles, Kakehi, Bangma.

Other (specify): None.

Financial disclosures: Leonard P. Bokhorst certifies that all conflicts of interest, including specific financial interests and relationships and affiliations relevant to the subject matter or materials discussed in the manuscript (eg, employment/affiliation, grants or funding, consultancies, honoraria, stock ownership or options, expert testimony, royalties, or patents filed, received, or pending), are the following: None.

Funding/Support and role of the sponsor: The PRIAS study is supported by grants from the Prostate Cancer Research Foundation (SWOP), Rotterdam.

Acknowledgment statement: The authors acknowledge the PRIAS study group for contributing to the acquisition of the data.

\section{Appendix A. Supplementary data}

Supplementary data associated with this article can be found, in the online version, at http://dx.doi.org/10.1016/j. eururo.2016.06.007.

\section{References}

[1] van den Bergh RC, Roemeling S, Roobol MJ, Roobol W, Schroder FH, Bangma $\mathrm{CH}$. Prospective validation of active surveillance in prostate cancer: the PRIAS study. Eur Urol 2007;52:1560-3.

[2] van den Bergh RC, Vasarainen H, van der Poel HG, et al. Shortterm outcomes of the prospective multicentre 'Prostate Cancer Research International: Active Surveillance' study. BJU Int 2010; 105:956-62.

[3] Bul M, Zhu X, Valdagni R, et al. Active surveillance for low-risk prostate cancer worldwide: the PRIAS study. Eur Urol 2012;63: 597-603.

[4] Bokhorst LP, Alberts AR, Rannikko A, et al. Compliance rates with the Prostate Cancer Research International Active Surveillance (PRIAS) protocol and disease reclassification in noncompliers. Eur Urol 2015;68:814-21.
[5] Bul M, Zhu X, Rannikko A, et al. Radical prostatectomy for low-risk prostate cancer following initial active surveillance: results from a prospective observational study. Eur Urol 2012;62:195-200.

[6] Kweldam CF, Wildhagen MF, Bangma CH, van Leenders GJ. Diseasespecific death and metastasis do not occur in patients with Gleason score $<\mid=6$ at radical prostatectomy. BJU Int 2015;116:230-5.

[7] Ross HM, Kryvenko ON, Cowan JE, Simko JP, Wheeler TM, Epstein JI. Do adenocarcinomas of the prostate with Gleason score (GS) $<=6$ have the potential to metastasize to lymph nodes? Am J Surg Pathol 2012;36:1346-52.

[8] Klotz L, Vesprini D, Sethukavalan P, et al. Long-term follow-up of a large active surveillance cohort of patients with prostate cancer. J Clin Oncol 2015;33:272-7.

[9] Tosoian JJ, Mamawala M, Epstein JI, et al. Intermediate and longerterm outcomes from a prospective active-surveillance program for favorable-risk prostate cancer. J Clin Oncol 2015;33:3379-85.

[10] Welty CJ, Cowan JE, Nguyen H, et al. Extended follow-up and risk factors for disease reclassification from a large active surveillance cohort for localized prostate cancer. J Urol 2015;193:807-11.

[11] Schoots IG, Petrides N, Giganti F, et al. Magnetic resonance imaging in active surveillance of prostate cancer: a systematic review. Eur Urol 2014;67:627-36.

[12] Hoeks CM, Somford DM, van Oort IM, et al. Value of 3-T multiparametric magnetic resonance imaging and magnetic resonanceguided biopsy for early risk restratification in active surveillance of low-risk prostate cancer: a prospective multicenter cohort study. Invest Radiol 2014;49:165-72.

[13] Walton Diaz A, Shakir NA, George AK, et al. Use of serial multiparametric magnetic resonance imaging in the management of patients with prostate cancer on active surveillance. Urol Oncol 2015;33:202 e1-2027e.

[14] Schoots IG, Roobol MJ, Nieboer D, Bangma CH, Steyerberg EW, Hunink MG. Magnetic resonance imaging-targeted biopsy may enhance the diagnostic accuracy of significant prostate cancer detection compared to standard transrectal ultrasound-guided biopsy: a systematic review and meta-analysis. Eur Urol 2015;68: 438-50.

[15] Moore CM, Parker C. The evolution of active surveillance for prostate cancer. Eur Urol 2015;68:822-3.

[16] Bokhorst LP, Lepisto I, Kakehi Y, et al. Complications after prostate biopsies in men on active surveillance and its effect on receiving further biopsies in the Prostate cancer Research International: Active Surveillance (PRIAS) study. BJU Int 2016;118:366-71.

[17] Bokhorst L, Alberts A, Kakehi Y, et al. Frequency of PSA testing in men on active surveillance for prostate cancer. J Urol 2015;193:e755.

[18] Bul M, van den Bergh RC, Rannikko A, et al. Predictors of unfavourable repeat biopsy results in men participating in a prospective active surveillance program. Eur Urol 2012;61:370-7.

[19] Ankerst DP, Xia J, Thompson Jr IM, et al. Precision medicine in active surveillance for prostate cancer: development of the CanaryEarly Detection Research Network active surveillance biopsy risk calculator. Eur Urol 2015;68:1083-8.

[20] Loeb S, Bruinsma SM, Nicholson J, et al. Active surveillance for prostate cancer: a systematic review of clinicopathologic variables and biomarkers for risk stratification. Eur Urol 2015;67:619-26.

[21] Bruinsma SM, Bangma CH, Obbink H, Roobol MJ. Active surveillance for low risk prostate cancer: the study protocol of the Movember Global Action Plan 3 (GAP3) project [abstract]. Eur Urol Suppl 2015;14:e1036-136. 